Begriff der absentia bestimmt werden: hier war das ganz besonders nöthig. Denn abesse heisst mehrfach gerade a tribunali abesse ${ }^{1}$ ). Das ist es aber hier nicht. So hat denn Ulpian den Ausdruck auch im 39. B. zum Sabin bei der Lehre von der Vormundschaft erläutert ${ }^{2}$ ). Möglicherweise hat Ulpian hier auch von Ablehnung und 'Nennung des Geeigneteren' gesprochen. Dann wäre es klar, wie diese ganze Lehre als Tribunalssache in unser Werk käme. U. hatte freilich beides schon anderswo weitläufig abgehandelt; aber vor Wiederholungen konnte sich seine praktische Schriftstellerei nicht scheuen.

Nach allem dem scheinen mir die uns aufbehaltenen Bruchstücke des Werkes sich dem Plane, wie ich ihn entworfen habe, ganz gut und ohne grosse Schwierigkeit einzufügen.

\title{
VIII.
}

\section{Ueber die Handschrift Nr. 82 des Domcapitels zu Vich.}

\author{
Von \\ Herrn Geh. Justizrath Professor Dr. Fitting \\ in Halle.
}

In Savigny's Geschichte des römischen Rechts im MA. 2. Ausg. IV. S. 220 ist unter den Handschriften der Summa Codicis des Rogerius auch eine Handschrift der Dombibliothek zu Vich (in Catalonien) verzeichnet mit Verweisung auf Serapeum 1847. 93. N. 82 $^{3}$ ). Auch Denifle, Die Universitäten des MA. bis 1400 Bd. I S. 158 Anm. 391 erwähnt diese Hand-

1) fr. 51 \$5 de fcomm. lib. 40,5 ; fr. $4 \$ 5$ de da. inf. 39,2 . $\left.{ }^{2}\right)$ fr. $173 \S 1$ de VS. $-{ }^{3}$ ) Im Serapeum von $1847 \mathrm{~S} .90$ ff. steht nämlich ein Verzeichniss der Handschriften der Bibliothek der Kathedrale zu Vich von Dr. G. Heine, worin es S. 93 heisst: „,82. Summae institutionum a Placentino compositae apud Montem Pessulanum. Ej. legum summae. Summae Trogerii super codice Iustiniani. mbr. sc. 13.“ 
schrift mit den Worten: "Summa Rogers - - zu Vich in Spanien (Capitelsbibl. n. 82 nach den Summen Placentins)“.

Da es mir darauf ankam bestimmt zu wissen, ob die Handschrift wirklich die Summa des Rogerius oder die mit ihr eng verwandte Summa Trecensis enthalte, so wendete ich mich nach Vich mit der Bitte um die Mittheilung der Anfangsund der Schlusswörter der Summa. Als Antwort erhielt ich nicht bloss die erbetene Auskunft; sondern zufolge des nicht dankbar genug zu erkennenden gütigen Entgegenkommens des Hochwürdigsten Bischofs, Dr. D. José Morgades y Gili, sowie des Canonicus und Archivars des Domcapitels, Dr. D. Jaime Serra y Jordi, besonders aber zufolge der ausnehmenden Gefälligkeit des Secretärs des bischöflichen archäologischartistischen Museums, Herrn José Serra y Campdelacreu, besitze ich jetzt eine von dem Letzteren verfertigte vollständige und sehr genaue Beschreibung der ganzen Handschrift. Diese Beschreibung ist um so werthvoller und schätzbarer, je weniger die in Spanien befindlichen Handschriften bekannt sind. Ich glaube daher meinen Dank für die mir bewiesene grosse Liberalität nicht besser bethätigen zu können als dadurch, dass ich die mir gewordenen Mittheilungen in dieser Zeitschrift veröffentliche und sie so zum Gemeingut aller Derjenigen mache, die für solche Dinge ein Interesse haben.

Die Anmerkungen rühren sämmtlich von mir her.

Die Handschrift - Codice $\mathrm{n}^{\circ} .82$ del Archivo Capitular de la Catedral de Vich - ist Eigenthum des Domcapitels zu Vich, befindet sich aber gegenwärtig in der bibliographischen Abtheilung des dortigen bischöflichen archäologisch-artistischen Museums. Dieses Museum wurde im Jahre 1890 von dem obengenannten Bischof, dem Protector der Wissenschaft, Litteratur und Kunst, gegründet und eröffnet. Gleich von Anfang sehr reich und bedeutend, hat es an Gegenständen des christlichen Mittelalters seines Gleichen nicht in Spanien, an Tafelgemälden vom 9.-12. Jahrhundert nach der Versicherung der Sachverständigen vielleicht nicht in ganz Europa.

Die Handschrift, in Holzdeckel mit Lederüberzug gebunden, ist mit diesem Einbande gemessen 27,5 Centim. hoch, 
Fitting,

19,5 Centim. breit und 7 Centim. dick. Sie trägt folgende gedruckte Signatur aus neuer Zeit:

LXXXII - Placentini summæ - institutionum.

Sie besteht im Ganzen aus 252 nicht numerirten Pergamentblättern in $4^{0}$, zweispaltig geschrieben. Die Schrift, welche für den ganzen Inhalt des Bandes von einer und derselben Hand herrühren kann, misst der Höhe nach im Allgemeinen 1 Millim. Sie ist mit zahlreichen Initialen verziert, die zwar meist einfach, aber sämmtlich sehr hübsch sind, besonders diejenigen, die in den Rand hineinragen und sich dort zu Palmetten entfalten. Zur Verzierung sind nur Roth und Blau verwendet. Die Schrift weist viele Abbreviaturen auf. Sie ist dem 13. Jahrhundert zuzutheilen. ${ }^{1}$ )

Bl. 1 steht oben am Rande:

hic liber est sancti petri vici.

Z. 1 folgt dann in Roth:

Incipiunt summe institutionum. A placentino composite apud montem pessulanum ad euidentiam iuditium (sic).

und hierauf in Schwarz der Text der Summa:

Iuris arti professionique boni et equi cunctos literatos e (leg. literatorie) scientie amatores convenit infudare (leg. insudare) expedit inuigilare. decet opera (leg. operam) dare ${ }^{2}$ )

und so fort bis zum Ende von Bl. 40, wo zufolge einer Verheftung der Pergamentlagen der Text der Summa mit den Silben „reli“ abbricht, um sich erst Bl. 235 mit den Silben "giosis" fortzusetzen. Spalte 4 Zeile 5 dieses Blattes endigt die Summa und beginnt ein neues Werk mit folgenden Vermerken in Roth:

Explicit summa institutionum a pla. edita. Incipit tractatus onnium accionum ab eodem compositus.

Der Uebergang zu diesem Tractat ist weder durch Beginn einer neuen Zeile, noch sclbst nur durch eine grössere

1) Dieser Ansicht bin auch ich nach den der Beschreibung einverleibten sorgfältig facsimilirten Proben. Doch möchte ich danach die Schrift in eine frülse Zeit des 13. Jahıh. selzen. -- - ) Die ganze, in den Druckausgaben der Summa fehlende, Einleitung findet sich in meinen Juristischen Schriften des früheren Mittelalters S. 217 if. 
Initiale hervorgehoben; die (tilgenden) Punkte unter ,omnium“" stehen in der Handschrift.

Der Tractat reicht bis Bl. 250 Sp. 2, wo er Z. 4 mit den zu dem Titel „de exercitoria accione" gehörigen Wörtern „qualitate negocii et accionis genere" endigt. ${ }^{1}$ ) Der Rest der Spalte ist unbeschrieben.

Bl. 250 Sp. 3 K. 1 folgt mit der Ueberschrift (in Roth) „de hostiariis" ein Capitel dieses Inhaltes, dann entsprechend Capitel „de lectoribus", „de exorcistis" etc. bis zu dem Capitel "de sacris ordinibus", welches am Ende der letzten Spalte von Bl. 252 mit den Wörtern ,a $\mathrm{x}^{0}$ judicari for" abbricht. Hiermit endigt aber nicht bloss dieser (canonistische) Tractat, sondern zugleich der ganze Band und die falsch eingeheftete Pergamentlage, die nach $\mathrm{Bl}, 40$ hätte folgen sollen. Die Beschreibung muss daher zu diesem Blatte zurückkehren.

Bl. 41 ist leer und sogar ohne Linien. Bl. 42 Sp. 1 Z. 1 beginnt die Summa Codicis des Placentinus mit den folgenden Versen:

$[H]$ as legum summas, si quis uult iura tueri, legat (leg. perlegat), et sapiens si uult orator haberi.

$[H]$ oc placentinus tibi docta bononia munus [g]ratum causidicis utile mittit opus. ${ }^{2}$ )

Hierauf in grossen, reich verzierten (in Facsimile wiedergegebenen) Initialen als besondere Zeile die beiden ersten Wörter des Prologs

\section{UONDAM EGO}

(für das fehlende $Q$ ist reichlich Raum freigelassen) und sodann in gewöhnlicher Schrift weiter ego credidi quod sufficent (leg. sufficeret) satisque

1) Die Handschrift enthält also die bei Saviguy IV, §§ 78,79 (S. 209 ff.) beharndelte Schrift des Placentinus, welche in den Druckausgaben und in der Vaticanischen Handschrift den Titel führt: „De varietate actionum", und zwar soweit sie von Placentinus herrührt, d. h. bis zum Ende des zweiten Buches. -- 2) Diese Verse stehen auch in allen übrigen mir bekannten Handschriften der Sumna: der Frankfurter, der Wiener und der Lejpziger (s. meine Juristischen Schriften des früheren MA. S. 24 ff.). Bei Savigny IV. Anhang XIV (S. 542) sind sie nicht mit ahgedruckt und nicht einmal (S. $270 \mathrm{ff}$.) in seiner Beschreibung der Summa und ihrer Handschriften erwähnt. 
michi memoriale foret, si opus frotgerii $\left.{ }^{1}\right)$ inperfectum fecissem (leg. perfecissem), si codicis summas ab eodem initiatas competenti medio fineque congruo conclusissem. etc. ${ }^{2}$.

Dieser Prolog füllt die ganze Spalte 1 und einen Theil der Spalte 2. Dann folgt die (bisher ungedruckte) Einleitung der Summa:

Exponamus itaque quo nomine liber sacrarum constitutionum nuncupetur etc.

Die Summa bricht Bl. $117 \mathrm{Sp} .4$ in der Mitte der letzten Zeile ab mit den Wörtern:

ut manumittatur manumittere non uoluit. $\dot{v}$. D. de manumissionibus. Lege. Secunda;.$^{3}$ )

Bl. 118 Sp. 1 Z. 1 in rother Schrift:

Incipiunt summe frogerii super codicem iustiniani und nun in gewöhnlicher Schrift der Prolog:

Cum multe esent partes iuris ciuilis que confuse per diuersa uolumina etc.

Bl. 224 letzte Zeile der Sp. 3:

Explicit liber. VIII. Incipit. IX. qui accusari non possunt.

worauf, nach einigen leeren Zeilen am Ende der Sp. 3, mit Sp. 4 der Text von IX, 1 beginnt.

Bl. 234 Sp. 2 endigt ein wenig unter der Mitte der Spalte der Titel „De sententiam passis et restitutis“ und zugleich die ganze Summa mit den Wörtern:

omnia adimit. sic et restitutio etiam simpliciter facta omnia restituit. v. c. e. l. vlt. ${ }^{4}$ )

1) Diese Form weist nach gefälliger Mittheilung meines Collegen, Herrn Prof. Suchier, auf provençalische Herkunft der Handschrift hin. Denn „Rotgier" mit tg ist die gewöhnliche provençalische Schreibung. Die Catalanen (Ramon Muntaner, Andreu Febrer) schreiben „Roger". 2) Der Prolog ist bei Savigny IV. Anhang XIV. (S. 542 fg.) vollständig abgedruckt. - $\left.{ }^{3}\right)$ Die Summa scheint also in der Handschrift nur bis zu Titel VII, 1 zu reichen. In der Druckausgabe der Summa steht freilich im genannten Titel eine solche Stelle nicht. - ${ }^{4}$ ) In der Handschrift steht also die Summa vollständig. Jedoch entsprechen die Schlussworte nicht denjenigen der Summa in der Tübinger Handschrift; es sind vielmehr djejenigen der Summa Godicis des Placentinus. Demnach scheint hier die Summa des Rogerius nicht in der ursprünglichen Ge- 
Das Werk des Rogerius beginnt mit sehr schwarzer Tinte, die aber allmählich blässer wird, so dass sich am Schlusse auf einigen Seiten die Farbe der Buchstaben kaum von dem gelblichen Pergament abhebt.

Auf jene Schlusswörter der Summa folgt eine leere Zeile und sodann mit sehr schwarzer, stark gegen die vorhergehende abstechender Schrift:

$[S]_{\text {I }}$ PACTO quo penam. actum (leg. pactum) nudum processit. puta de scribendo codice etc.

Dieses Stück endigt B1. 234 Sp. 4 etwas unter der Mitte der Spalte mit den Wörtern:

aūtt. et D. de arbitris. l. vlt. Plac̄. ${ }^{1}$ )

Der Inhalt der Blätter $235-252$ ist oben schon angegeben.

\title{
IX. \\ Beiträge zur Kenntniss und Würdigung des sogen. westgothischen Gaius.
}

\author{
Von \\ Herrn Dr. H. F. Hitzig,
}

Docenten in Zürich.

Dass der sogenannte westgothische Gaius nicht eine erst von den Redactoren des Breviars verfasste, sondern eine bereits früher entstandene und von den Redactoren aufgenommene Gaiusbearbeitung darstellt, dürfte heute kaum mehr bezweifelt werden; den Ausführungen Dernburgs ${ }^{2}$ ) (Institutionen des

stalt, sondern mit der Fortsetzung und Ueberarbeitung des Placentinus vorzuliegen, über welche ich mich bei anderer Gelegenheit äussern werde.

1) Wir besitzen also hier die bei Savigny IV. $\$ 86$ E. (S. 283) verzeichnete Summa des Placentinus zu der L. Si pacto quo poenam (L. 14 C. de pact. II, 3), welche Savigny nur aus einem Citat in Placentins Summa Codicis (VIII, 41 in f.) bekannt war. - ${ }^{2}$ ) Dazu die Besprechung von Degenkolb i. d. kritischen Vierteljahrsschrift XIV p. $503 \mathrm{ff}$. 